\title{
Screening of Bioactivity in Extracts from Different Varieties of Lettuce ${ }^{\dagger}$
}

\author{
Filomena Monica Vella ${ }^{1}$, Roberto Calandrelli ${ }^{1}$, Domenico Cautela ${ }^{2}$ and Bruna Laratta ${ }^{1, *}$ \\ 1 National Research Council (CNR), Institute of Research on Terrestrial Ecosystems (IRET), via P. Castellino, \\ 111,80131 Naples, Italy; filomenamonica.vella@cnr.it (F.M.V.); roberto.calandrelli@cnr.it (R.C.) \\ 2 Experimental Station for the Industry of the Essential Oils and Citrus Products (SSEA) - Special Agency of \\ the Chamber of Commerce of Reggio Calabria, via T. Campanella, 12, 89125 Reggio Calabria, Italy; \\ dcautela@ssea.it \\ * Correspondence: bruna.laratta@cnr.it \\ + Presented at the 1st International Electronic Conference on Plant Science, 1-15 December 2020; Available \\ online: https://iecps2020.sciforum.net/.
}

Published: 1 December 2020

\begin{abstract}
Lettuce (Lactuca sativa L.) belongs to Asteraceae family and is a widely grown and a popularly consumed vegetable worldwide cause leafy vegetables are common items in a wellbalanced diet. Lettuce is low in calories, fat and sodium. It is a good source of fiber, iron, folate, vitamin $C$ and various bioactive compounds. Lettuce is consumed in salad mixes and, as its consumption is increasing, is becoming one of most 'healthy' food. In vitro and in vivo studies have shown anti-inflammatory, cholesterol-lowering, anti-diabetic, and antioxidant activities attributed to the bioactive compounds in lettuce. The healthy properties are attributed to a large supply of antioxidant compounds mainly polyphenols. As they act as free radical scavengers, both these secondary plant metabolites are fundamental to counter oxidative stress, inflammation, cancer, diabetes, age-related neurodegeneration and cardiovascular disease. Further, various studies have investigated the effects of the polyphenolic compounds present in green lettuce, in reducing oxidative and anti-inflammatory stresses. This work aims to assess polyphenolic content, as well as related antioxidant capacity of 16 lettuce types, belonging to butterhead (L. capitata) and crisphead (L. crispa) varieties, in order to know their potential correlation between phenolic content and antioxidant activity. Results have shown lattuce an interesting and cheap source of antioxidant phenolics as alternative natural compounds useful to retard oxidation in different food systems.
\end{abstract}

Keywords: lettuce; lactuca sativa; polyphenols; antioxidant activity

\section{Introduction}

Several pieces of scientific evidence promote a balanced, health-promoting type of diet, based on vegetables and fruit, as the basis to prevent the starting of chronic and age-related diseases [1-4].

The beneficial effects of fruits and vegetables are believed due to the presence of biomecules with healthy properties such as polyphenols that, with their antioxidant activity, play an important role in counteing oxidative stress and in protecting human body by related demage such as, inflammation, cancer, diabetes, age-related neurodegeneration, and cardiovascular diseases [5-8].

Among vegetables, lettuce (Lactuca sativa L.), which belong to the Asteraceae family, is one of the most widely grown and popularly consumed worldwide. The largest producer in the world is China, followed by the USA and Europe, that contribute about $57 \%, 14 \%$ and $11 \%$ of the total lettuce production, respectively [9]. In 2018, Italy was responsible of production of about 770,000 ton, which represented the $26 \%$ of European lettuce production [9]. 
Lettuce comes in a array of colors, sizes, and shapes and because of this variability it can be grouped by their types. According to Mou classification [10], there are six main lettuce groups based upon leaf shape, size, texture, head formation, and stem type: crisphead (var. crispa), butterhead (var. capitata), romaine or cos (var. longifolia), leaf or cutting (var. acephala), stem or stalk (var. angustana), and Latin lettuce (no scientific name).

Lettuce is popularly consumed as fresh product in salad mixes and nowadays consumption of salads is increasing, above all for to the consumer perception of healthy food [11]. Moreover, since lettuce is generally eaten raw, more nutrients and bioactive compounds are retained compared to other vegetables that are cooked or processed, avoiding in this way the degradation of the thermolabile phytochemicals with potential nutraceutical interest $[8,12]$. Various studies have investigated the effects of the polyphenolic compounds present in lettuce, in reducing oxidative and anti-inflammatory stresses [11-13].

The objective of this study was to provide information about the potential use of lettuce as an effective source of natural antioxidants occurring naturally in foods; specifically 16 lettuce, belonging to butterhead (var. capitata) and crisphead (var. crispa) types were compare with respect to its contents of polyphenols and antioxidant activity, with the aim to determine the lettuce type that provides the greatest health-beneficial bioactive compounds.

\section{Experiments}

\subsection{Reagents and Standards}

All reagents and solvents were of analytical grade or otherwise stated. Gallic acid standard and 2,2-diphenyl-1-picrylhydrazyl (DPPH) were purchased from Sigma Chemical Co. (St. Louis, MO, USA).

\subsection{Plant Material and Sample Extraction}

In this study 16 lettuce, belonging to butterhead (var. capitata) and crisphead (var. crispa) varieties were hand-harvested in Villa Literno (Caserta province, Italy; $41^{\circ} 0^{\prime} 33 \mathrm{~N}-14^{\circ} 4^{\prime} 34 \mathrm{E}$ ) at commercial maturity condition.

For each plant, outer leaves (second stage of leaves) were detached and lightly washed with distilled water to remove eventual residues. Five grams of leaf were placed in an extracting solution of $49 \mathrm{~mL}$ methanol and $1 \mathrm{~mL} \mathrm{HCl} \mathrm{37 \%} \mathrm{[12].} \mathrm{The} \mathrm{solution} \mathrm{was} \mathrm{covered} \mathrm{to} \mathrm{avoid} \mathrm{evaporation,} \mathrm{shaken}$ at $100 \mathrm{rpm}$ at $20^{\circ} \mathrm{C}$ in the dark for $2 \mathrm{~h}$. The resulting extracts were filtered through Whatman paper filter, dried using a rotary evaporator (IKA RV8) and immediately stored at $-20{ }^{\circ} \mathrm{C}$, until analyses were performed.

\subsection{Total Polyphenols Content}

The total phenolics content was determined by the Folin-Ciocalteu assay [14]. Briefly, different aliquots of extracts were diluted to a $150 \mu \mathrm{L}$ final volume with distilled deionized water. FolinCiocalteu's reagent $(750 \mu \mathrm{L})$ and $600 \mu \mathrm{L}$ of $7.5 \% \mathrm{Na}_{2} \mathrm{CO}_{3}$ were added to the sample. The absorbance at $765 \mathrm{~nm}$ was read after incubation in the dark for $2 \mathrm{~h}$ at room temperature. Total phenolics content was expressed as mg of gallic acid equivalents (GAE) per g of fresh weight (FW). Samples were analysed in triplicate.

\subsection{In Vitro Antioxidant Activity}

The antioxidant activity of lettuce extracts was evaluated the DPPH (2,2-diphenyl-1picrylhydrazyl) radical scavenging assay according Blois procedure [15]. Briefly, $1.35 \mathrm{~mL}$ of $60 \mu \mathrm{M}$ DPPH radical in methanol were added to different extract concentrations, ranging from 10 to 1000 $\mu \mathrm{g} / \mathrm{mL}$. The decrease in absorbance at $517 \mathrm{~nm}$ was continuously determined until absorbance stabilization. The radical scavenging activity percentage (\%RSA) of DPPH discoloration was calculated according to the formula: 


$$
\% \text { RSA }=((\text { A_DPPH-A_s })) / \text { A_DPPH } \times 100
$$

where AS was the absorbance of the solution when the essential oil was added and ADPPH was the absorbance of the DPPH solution. The extract concentration (EC) necessary to achieve a $50 \%$ of radical DPPH inhibition (EC50) was obtained by plotting the RSA percentage as function of extract concentrations and was expressed as $\mathrm{mg} / \mathrm{mL}$.

\subsection{Statistical Analyses}

Samples were analyzed in triplicates and all results were expressed as mean \pm standard deviation (SD). Means, SD, calibration curves and linear regression analyses (R2) were determined using Microsoft Excel 2013 (Microsoft Corporation, Redmond, WA, USA). Correlation analyses were carried out by using CORREL function in Microsoft Excel 2013. Pearson correlation coefficients (r) were calculated and followed by t-Student test, with two-sample equal variance and two-tailed distribution. Differences at $p<0.05$ were considered significant and $p<0.01$ were highly significant.

\section{Results and Discussion}

Phenolic compounds refer to an important group of plant secondary metabolites responsible for the plant's defense system. More than 8000 polyphenols have been identified in all plant organs [7] and affect the sensory and nutritional properties of plant-based foods [16]. Polyphenols have been described to have greater antioxidant activity than vitamins C and E [17]. Individual phenolic compounds and vegetables that contain phenolic compounds have shown beneficial effects against oxidative stress, inflammation, cancer, diabetes, age-related neurodegeneration, and cardiovascular diseases [5-8].

In this study 8 butterhead (code number $6,7,10,11,12,13,14$, and 15) and 8 crisphead (code number 16, 17, 18, 19, 20, 21, 22, and 23) lettuces were analyzed considering both total polyphenols content and antioxidant activity. The results were reported in Table 1 and in Figure 1, respectively.

Total phenolic content in lettuce varies among lettuce types. In general, crisphead lettuces were on average lower in total phenolic content compared to butterhead types. Butterhead number 22 had the highest value of total polyphenols, as shown in Table 1.

Table 1. Poyphenols content (mg GAE/100 g FW) of lettuces.

\begin{tabular}{ccc}
\hline Lactuca sativa Type & Code Number & mg GAE/100 g FW * \\
\hline crisphead & 6 & $109.69 \pm 7.12$ \\
crisphead & 7 & $100.34 \pm 6.83$ \\
crisphead & 10 & $72.11 \pm 3.75$ \\
crisphead & 11 & $80.44 \pm 3.31$ \\
crisphead & 12 & $121.66 \pm 5.38$ \\
crisphead & 13 & $90.91 \pm 5.59$ \\
crisphead & 14 & $119.40 \pm 7.58$ \\
crisphead & 15 & $58.67 \pm 2.57$ \\
butterhead & 16 & $85.51 \pm 1.82$ \\
butterhead & 17 & $95.36 \pm 1.97$ \\
butterhead & 18 & $99.88 \pm 2.44$ \\
butterhead & 19 & $118.67 \pm 7.54$ \\
butterhead & 20 & $79.00 \pm 4.27$ \\
butterhead & 21 & $101.80 \pm 5.29$ \\
butterhead & 22 & $142.70 \pm 6.97$ \\
butterhead & 23 & 123.54 \\
\hline
\end{tabular}

* GAE = gallic acid equivalent; $F W=$ fresh weigth.

Differences in phenolic content of lettuce types could be due to genetic, agronomical and environmental factors that influence the chemical composition and in turn affect polyphenolic 
amount [11]. Particularly, the abundance of these compounds may be altered by many factors, such as light intensity (quality and quantity), water availability and irrigation regime, nutrient supply, use of pesticide, weather conditions, growing season as well as fertilization, production, cultivation, and storage procedures (Durazzo et al., 2014, Kim et al., 2016).

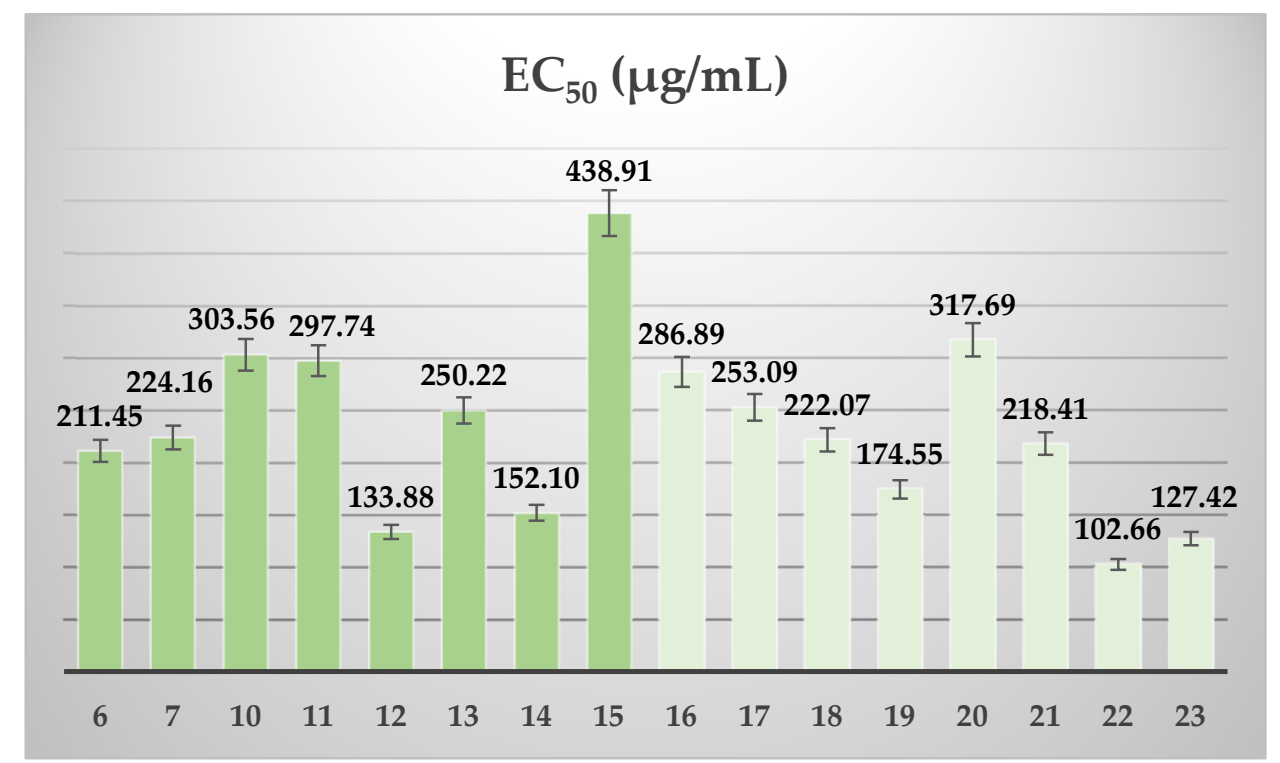

Figure 1. Antioxidant activity of lettuces expressed as EC50 $(\mu \mathrm{g} / \mathrm{mL})$. Green = crisphead; light green $=$ butterhead.

The results of DPPH radical scavenging activity was presented as EC50 values. As showed in Figure 1, butterhead number 22 possessed the lowest EC50. Moreover, a highly significant negative correlation $(p<0.01)$ existed between total polyphenols and antioxidant activity of lettuce extracts. As a low EC50 indicates a high antioxidant activity of a sample, this result means that an increase in bioactive compounds corresponded to a lower extract concentration necessary to achieve $50 \%$ of DPPH inhibition, thus suggesting a higher antioxidant activity.

The DPPH radical scavenging activity is greatly influenced by the total phenolic content of the lettuce samples. In particular, the variation in polyphenolic content causes a variation in DPPH radical scavenging of extracts of lettuce. As mentioned earlier, different geographical locations, environmental factors and postharverst handling could affect the total phenolic content that indirectly altered the antioxidant activity of the lettuce samples $[8,18]$.

\section{Conclusions}

Lettuce is high in water content $(95 \%)$ and low in calories, is characterized by dietary fiber, presence of several important dietary minerals (low amount of $\mathrm{Na}$ and a relatively good amount of $\mathrm{Fe}$ ), and various vitamins (folate and vitamins C).

Despite consumer expectations, lettuce can provide considerable amounts of healthy nutrients, particularly phenolic compounds. The results of this study demostrate that lettuce is rich in bioactive phytochemicals, thus increasing the nutritional value of lettuce in human diet. Notably, butterhead types were found to be slightly richer in polyphenols than crisp lettuce, hence recommending more the use of the former variety.

Further studies concerning the bioavailability of polyphenols in lettuce and their nutraceutical potential application are needed to evaluate the health-promoting benefits.

Author Contributions: Conceptualization, B.L. and F.M.V.; investigation, R.C., D.C., and F.M.V.; data analysis D.C., B.L. and F.M.V; writing-review and editing B.L. and F.M.V. All authors have read and agreed to the published version of the manuscript. 
Acknowledgments: The authors are grateful to Agostino Navarro for their helpful assistance during the sample preparation.

Conflicts of Interest: the authors declare no conflict of interest.

\section{References}

1. Hooper, L.; Cassidy, A. A review of the health care potential of bioactive compounds. J. Sci. Food Agric. 2006, 86, 1805-1813.

2. Patil, B.S.; Jayaprakasha, G.K.; Chidambara Murthy, K.N.; Vikram, A. Bioactive compounds: Historical perspectives, opportunities, and challenges. J. Agric. Food Chem. 2009, 57, 8142-8160.

3. Shashirekha, M.N.; Mallikarjuna, S.E.; Rajarathnam, S. Status of bioactive compounds in foods, with focus on fruits and vegetables. Crit. Rev. Food Sci. Nutr. 2015, 55, 1324-1339.

4. Altemimi, A.; Lakhssassi, N.; Baharlouei, A.; Watson, D.G., Lightfoot, D.A. Phytochemicals: Extraction, isolation, and identification of bioactive compounds from plant extracts. Plants 2017, 6, 42.

5. Hung, H.C.; Joshipura, K.J.; Jiang, R.; Hu, F.B.; Hunter, D.; Smith-Warner, S.A.; Colditz, G.A.; Rosner, B.; Spiegelman, D.; Willett, W.C. Fruit and vegetable intake and risk of major chronic disease. J. Natl. Cancer Inst. 2004, 96, 1577-1584.

6. Morris, M.C.; Evans, D.A.; Tangney, C.C.; Bienias, J.L.; Wilson, R.S. Associations of vegetable and fruit consumption with age-related cognitive change. Neurology 2006, 67, 1370-1376.

7. Dai, J.; Mumper, R.J. Plant phenolics: Extraction, analysis and their antioxidant and anticancer properties. Molecules 2010, 15, 7313-7352.

8. Kim, M.J.; Moon, Y.; Tou, J.C.; Mou, B.; Waterland, N.L. Nutritional value, bioactive compounds and health benefits of lettuce (Lactuca sativa L.). J. Food Compos. Anal. 2016, 49, 19-34.

9. Faostat, Food and Agriculture Organization of the United States. Available online: http://www.fao.org/home/en/ (accessed on 17 October 2020).

10. Mou, B. Nutritional quality of lettuce. Curr. Nutr. Food Sci. 2012, 8, 177-187.

11. Llorach, R.; Martinez-Sanchez, A.; Tomas-Barberan, F.A.; Gil, M.I.; Ferreres, F. Characterization of polyphenols and antioxidant properties of five lettuce varieties and escarole. Food Chem. 2008, 108, 10281038.

12. Adesso, S.; Pepe, G.; Sommella, E.; Manfra, M.; Scopa, A.; Sofo, A.; Tenore, G.C.; Russo, M.; Di Gaudio, F.; Autore, G.; et al. Anti-inflammatory and antioxidant activity of polyphenolic extracts from Lactuca sativa (var. Maravilla de Verano) under different farming methods. J. Sci. Food Agric. 2016, 96, 4194-4206.

13. Pepe, G.; Sommella, E.; Manfra, M.; De Nisco, M.; Tenore, G.C.; Scopa, A.; Sofo, A.; Marcozzo, S.; Adesso, S.; Novellino, T.; et al. Evaluation of anti-inflammatory activity and fast UHPLC-DAD-IT-TOF profiling of polyphenolic compounds extracted from green lettuce (Lactuca sativa L.; var. Maravilla de Verano). Food Chem. 2015, 167, 153-161.

14. Singleton, V.L.; Rossi, J.A., Jr. Colorimetry of total phenolics with phosphomolybdic-phosphotungstic acid reagents. Am. J. Enol. Vitic. 1965, 16, 144-158.

15. Blois, M.S. Antioxidant determinations by the use of a stable free radical. Nature 1958, 181, 1199-1200.

16. Karakaya, S. Bioavailability of phenolic compounds. Crit. Rev. Food Sci. Nutr. 2004, 44, 453-464.

17. Rice-Evans, C.A.; Miller, J.; Paganga, G. Antioxidant properties of phenolic compounds. Trends Plant Sci. 1997, 2, 152-159.

18. Durazzo, A.; Azzini, E.; Lazzè, M.C.; Raguzzini, A.; Pizzala, R.; Maiani, G.; Palomba, L.; Maiani, G. Antioxidants in Italian head lettuce (Lactuca Sativa var. capitata L.) grown in organic and conventional systems under greenhouse conditions. J. Food Biochem. 2014, 38, 56-61.

Publisher's Note: MDPI stays neutral with regard to jurisdictional claims in published maps and institutional affiliations.

(C) 2020 by the authors. Submitted for possible open access publication under the terms and conditions of the Creative Commons Attribution (CC BY) license (http://creativecommons.org/licenses/by/4.0/). 